\title{
Numerical analysis of flow in a modular heat exchanger used in food industrial plants
}

\author{
Wojciech Judt ${ }^{1, *}$, and Jarosław Bartoszewicz ${ }^{1}$ \\ 1 Poznan University of Technology, Chair of Thermal Engineering, Piotrowo 3 St., 60-965 Poznań, Poland
}

\begin{abstract}
In the article is proposed a construction of modular cross-flow heat exchanger. This construction can be used in food industrial plants for example to heating a palm oil for a chips production. The second possibility to make use of that heat exchanger in a food industrial plants will be a heating of a worn out rape oil after a technological process for a improvement of effective utilization in oil boiler. The main advantage of modular heat exchangers is the possibility of simple adjustment of heating power dependent on demand. It is possible by valve system, which can cut off subsequent sections of the heat exchanger from the plant when heat requirement is decreasing. During a numerical simulation was demonstrated, that an exhaust gases flow in a heat exchanger is not homogenous. In numerical analysis was obtained a real flow velocity distribution and temperature distribution for a full geometry of the designed object and for two subsequent section of the heat exchanger.
\end{abstract}

\section{Introduction}

The food industry is one of a big beneficiary of heat usage for a production process. The main problem of a production process on food plants is a controlling of a power in installed heat exchangers. During a production process, necessarily is a dosage of an amount of heat delivered. This phenomenon depends on different heat demand for other parts of a fabrication, during a production process.

Authors of a paper analyzed a heat transfer process for a palm oil heating in a food industrial plant for a chips production. For a heating medium in a designed heat exchanger was a waste heat used from a heating boiler, which is used for a steam generation in another process, which is realized in the industrial plant. This heating boiler reaches a nominal power equal to $4 \mathrm{MW}$. Estimated heating power, which can be recovered from exhaust gases, generated by heating boiler with that power is about a $150 \mathrm{~kW}$. The second possibility of heat exchanger application is heating an used palm oil after a production for a reprocessing process. Heating of used palm oil before a combustion will increase a efficiency of reprocessing. A scheme of a heat recovery plant is shown in figure 1.

Popular construction of heat exchanger for food industrial plants is a plate heat exchangers, but this type of heat exchangers is not popular for heat transfer between gaseous and liquid mediums [1]. The main advantage of a proposed solution of a heat exchanger structure is a modular construction. The second advantage of planned construction is a simplicity of a heat exchanger production. The assumption, that a heat exchanger will be not exposed to a corrosion environment allows making a construction from a carbon steel. It causes, that a production of the heat exchanger will be cheap. The modular structure allows for a heat transfer controlling, between heated and cooled medium.

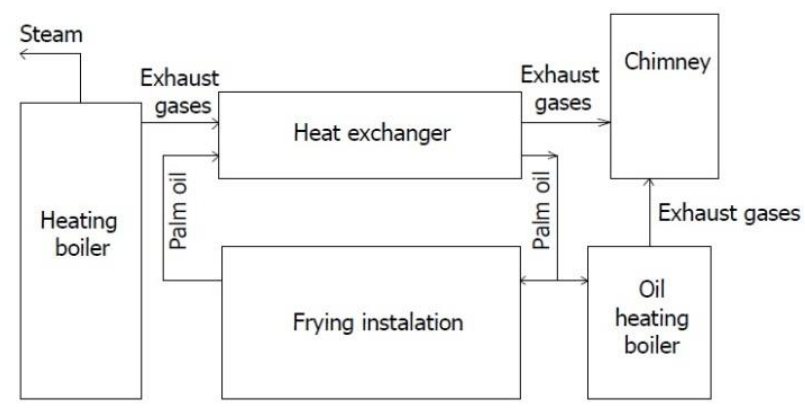

Fig. 1. Scheme of a heat recovery plant.

When a heat demand is lower, then possible is to decreasing an area of heat transfer. Heat power controlling can be controlled by valve set, which can be assembled after every module of the heat exchanger. The flow of a heated medium, which are flowing through pipes can be led out from the heat exchanger and a temperature of exhaust gases in the outlet of the construction can be controlled. This denouement causes that a risk of a heat exchanger damage by corrosion during a liquefaction of acids and water vapour is getting smaller. The phenomenon of a heat exchangers failure during a corrosion environment exposition is commonly present in improperly projected heat exchangers or heating boilers, wherein the coolest parts of heat exchangers is precipitated a liquid water or acids.

The main purpose of the paper is an analysis of exhaust gas real velocity and temperature distribution in

\footnotetext{
* Corresponding author: wojciech.judt@ put.poznan.pl
} 
a designed geometry of the heat exchanger. During a research was prepared a numerical analysis of a distribution of these parameters in the designed heat exchanger. Numerical analysis in a heat exchanger design process is a simple way for an estimation of basic parameters of a fluid flow, without a necessity of a prototype building or realization of experimental research. Numerical analysis is widely present in a research concerning with heat exchangers [2].

\section{Analysis and modeling}

Authors of article assumed, that a temperature of exhaust gases, which are leaving a heating boiler is around a $260{ }^{\circ} \mathrm{C}$. The minimal temperature of exhaust gases in the heat exchanger is conditioned by an avoiding of corrosion conditions when exhaust gases reach a temperature of water or acids condensation. The minimal temperature of exhaust gases in the outlet of the heat exchanger was assumed to equal a $180{ }^{\circ} \mathrm{C}$. The temperature of palm oil during a production process of a chips frying is equal to $180{ }^{\circ} \mathrm{C}$. For a palm oil returning from a production, line authors assumed, that a temperature at the inlet to the heat exchanger is equal to $130{ }^{\circ} \mathrm{C}$. A counter-current flow of the mediums will be realized through a heat exchanger. The mass flow of exhaust gases results from the amount of exhaust gases, which are generated from a combustion process in a heating boiler. The mass flow of combusted coal was computed from equation 1 .

$$
\dot{M}_{c}=\frac{\dot{Q}_{b}}{\eta_{b} \cdot W_{c}}
$$

It was assumed that efficiency of combustion process equal 0,8 . The low calorific value of combusted coal is equal $23 \mathrm{MJ} / \mathrm{kg}$. The obtained mass flow of combusted coal is equal to $0,2 \mathrm{~kg} / \mathrm{s}$. The mass flow of fumes, which was generated from the combustion process was computed from few simple equations [3] describing a quantity of generated mass flow of fumes from solid fuel combustion with combustion air factor equal to 1.8 , and known ultimate analysis of combusted coal. The calculated mass flow of exhaust gases is equal to 2.15 $\mathrm{kg} / \mathrm{s}$. The mass flow of palm oil is a result of a requisition of oil amount for a production process and can be variable. For a heat exchanger calculation assumed an average oil demand for a nominal production process and it equals to $1.3 \mathrm{~kg} / \mathrm{s}$.

The heat exchanger was designed in analytical calculations. After that was prepared a numerical simulation of heat exchanger working in the ANSYS Fluent environment. Numerical analysis was realized with the same thermodynamic parameters of fluids, as in analytical calculations. Authors of paper developed three numerical models. It was a numerical model of heat transfer for a first and second module of designed installation and analysis of two neighboring sections of one designed module of the heat exchanger.

\subsection{Analytical calculations}

Analytical calculations of the designed heat exchanger are based on the equation 2 which is describing a heat transfer by heat transmission

$$
\dot{Q_{e}}=h \cdot A \cdot \Delta t_{\log } .
$$

Overall heat transfer coefficient used for calculations is described by equation 3 [9]

$$
h=\frac{1}{\frac{d_{\text {ave }}}{h_{\text {c fumes }} \cdot d_{\text {in }}}+\frac{g_{\text {pipe }}}{k_{\text {pipe }}}+\frac{d_{\text {ave }}}{h_{\text {c oil }} \cdot d_{\text {ex }}}} .
$$

The log mean temperature difference is described by equation 4 [9]

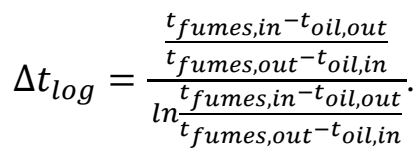

Log mean temperature difference is equal to $59.4{ }^{\circ} \mathrm{C}$. Thermodynamic parameters of fluids, which are exchanging heat in a heat exchanger were implemented for an average temperature of each medium. Parameters, which are needed for a realized calculation are presented in table $1[4,5]$.

Table 1. Thermodynamic parameters of a fluid, which are taking part in a heat transfer process.

\begin{tabular}{|c|c|c|}
\hline Parameter & Fumes [4] & Palm oil [5] \\
\hline $\mathrm{T}_{\text {ave }}\left[{ }^{\circ} \mathrm{C}\right]$ & 215 & 155 \\
\hline $\mathrm{Cp}[\mathrm{J} /(\mathrm{kg} \cdot \mathrm{K})]$ & 1097 & 2249 \\
\hline$\rho\left[\mathrm{kg} / \mathrm{m}^{3}\right]$ & 0,636 & 825,4 \\
\hline $\mathrm{k}[\mathrm{W} /(\mathrm{m} \cdot \mathrm{K})]$ & 0,04 & 0,16 \\
\hline $\operatorname{Pr}[-]$ & 0,65 & 47,7 \\
\hline$v\left[\mathrm{~m}^{2} / \mathrm{s}\right]$ & $3,28 \cdot 10^{-5}$ & $4,18 \cdot 10^{-6}$ \\
\hline
\end{tabular}

Heat transfer coefficient for exhaust gases was calculated by using a Nusselt number defined by Isaczenko in equation 5 [6]

$$
N u=0.26 R e^{0.65} \cdot P r_{f}^{0.33} \cdot\left(\frac{P r_{f}}{P r_{w}}\right)^{0.25} .
$$

Heat transfer coefficient for palm oil was calculated by using a Nusselt number defined by Sieder and Tate in equation $6[6]$

$$
N u=1.86\left(\operatorname{RePr} \frac{D}{L}\right)^{0.33} \cdot\left(\frac{\mu_{f}}{\mu_{w}}\right)^{0.14} .
$$

Results of analytical calculations for both mediums, which are transferring a heat are presented in table 2. 
Table 2. Results of analytical calculations of a heat transfer process in a designed heat exchanger.

\begin{tabular}{|c|c|c|}
\hline Parameter & Fumes & Palm oil \\
\hline $\operatorname{Re}[-]$ & 13400 & 400 \\
\hline $\mathrm{Nu}[-]$ & 86,2 & 81,4 \\
\hline $\mathrm{h}_{\mathrm{c}}\left[\mathrm{W} /\left(\mathrm{m}^{2} \cdot \mathrm{K}\right)\right]$ & 140 & 700 \\
\hline $\mathrm{h}\left[\mathrm{W} /\left(\mathrm{m}^{2} \cdot \mathrm{K}\right)\right]$ & \multicolumn{2}{|c|}{96} \\
\hline$\dot{Q}[\mathrm{~kW}]$ & \multicolumn{2}{|c|}{140} \\
\hline
\end{tabular}

Obtained power of designed heat exchanger during analytical calculations is close to assumed heat waste from exhaust gases, which can be recovered. Area of heat transfer obtained for designed heat exchanger during analytical calculations is equal to $24 \mathrm{~m}^{2}$.

Installation of the heat exchanger is built from two modules. Palm oil is located inside the pipes of the heat exchanger. Exhaust gases are flowing around the bank of tubes at the right angle to them. Each module has 2,5 meters length and is composed of five sections. After each section of the heat exchanger, flow of oil through pipes can be stopped and can be led out from installation. This solution allows for maneuvering a heat exchanger power. Each part of the heat exchanger is connected serially to next section.

\subsection{Numerical analysis of the full geometry of heat exchanger module}

A numerical model was implemented as a steady state. It is caused, because the main target of a paper is showing a character of a fluid flow in the designed heat exchanger, in steady state conditions. Numerical analysis was divided into two different models, each for the different module of the designed heat exchanger. Initially, calculations were realized for the first module of heat exchanger construction. After that, results obtained for a first module were implemented to a second model, as a boundary condition. The composition of results from two numerical models shows a whole distribution of flow and heat transfer parameters. Because of a model is a three-dimensional, analysis of a fluid flow requires solving a three equation of a flow velocity and a continuity equation. The turbulence of a flow in a heat exchanger was solved by a k-Epsilon turbulence model implementation.

For a heat transfer modeling, energy equation is needed to be solved. Heat transfer process in a numerical model is realized as an interface between two domains, representing exhaust gas and palm oil location in a heat exchanger volume. Thermal conditions of realized heat transfer are calculated via system coupling. Diaphragm influence for a heat transfer process is implemented to a model in the interface as a steel wall with $2,6 \mathrm{~mm}$ thickness. Exterior walls of the heat exchanger are implemented to a model as adiabatic.

\subsection{Numerical analysis of two sections of heat exchanger module}

Numerical analysis of two neighboring sections was realized for a third and fourth section, counting from a heat exchanger inlet. Two sections of the heat exchanger were analyzed in the detached model because this part is a periodical element of the designed heat exchanger. This analysis can better imitate a character of a fluid flow and heat transfer process, because a grid implemented for this model can be more detailed, as in a simulation for a whole module. Boundary conditions for this model originate from a numerical analysis for the first module of the designed heat exchanger. The boundary condition for the inlet of that module was implemented by a temperature and velocity distribution as a profile function in inlet plane to the modeling section.

\section{Results and discussion}

Figure 2 shows a temperature distribution for exhaust gases of the first module of designed installation, in a plane located between middle rows of pipes.

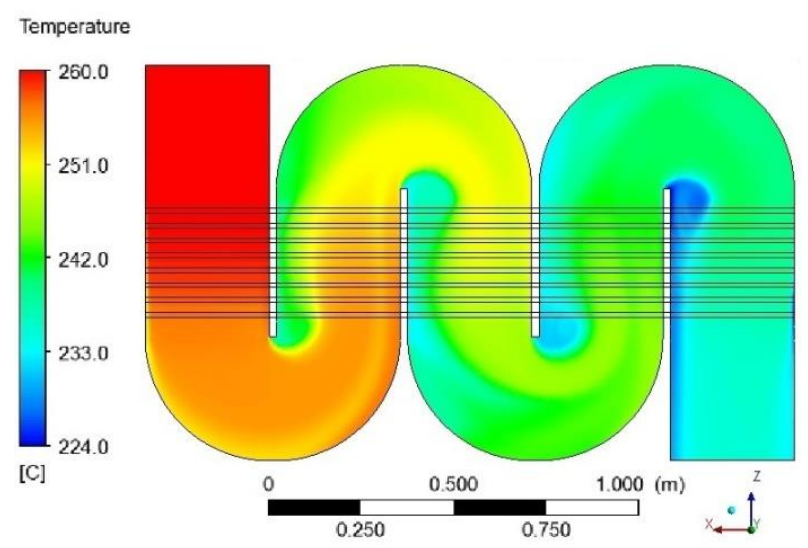

Fig. 2. The temperature distribution of exhaust gases for a first module of the heat exchanger.

Figure 3 shows a temperature distribution for exhaust gas of the second module in a plane located according to the middle row of pipes in the heat exchanger.

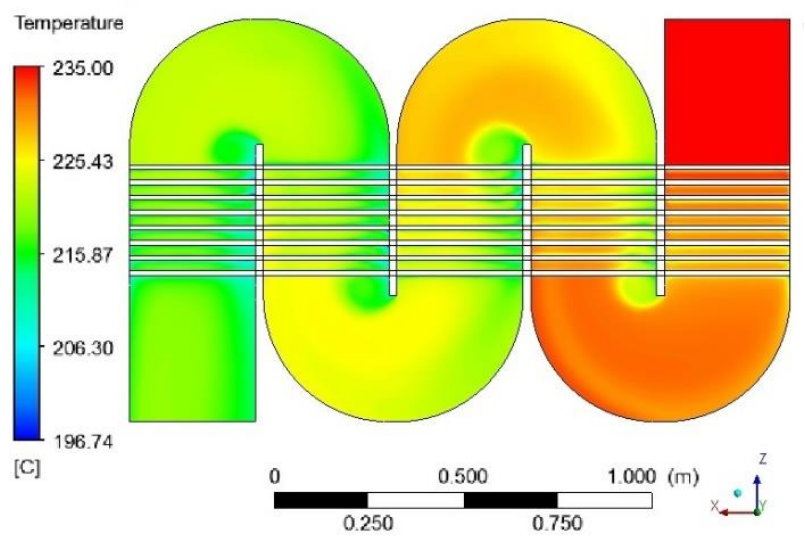

Fig. 3. The temperature distribution of exhaust gases in a second module of the heat exchanger. 
Numerical analysis of a temperature distribution for exhaust gases in whole heat exchanger geometry shows, that an average temperature in the outlet of the heat exchanger is equal to $215^{\circ} \mathrm{C}$. It means that a temperature difference between inlet and outlet of the heat exchanger is less than the assumption in analytical calculations. This phenomenon has an influence on a heat exchanger power. Obtained during numerical analysis power of heat exchanger is equal to $106 \mathrm{~kW}$ and is to $25 \%$ less than a power obtained from analytical calculations. This difference is a result of the accuracy of used equations for a Nusselt number. Increasing a power of heat exchanger requires the extension of a heat transfer area. This operation will increase costs of heat exchanger construction. Also, an extension of heat transfer area can expose a larger area of heat exchanger walls for a cooling under corrosion conditions. The minimal temperature of exhaust gases for designed heat exchanger construction is equal to $196,7{ }^{\circ} \mathrm{C}$. This temperature is less than an average temperature in the outlet of the heat exchanger but is not achieve a temperature of water or acids liquefaction.

Numerical analysis of an exhaust gases flows through a heat exchanger shows, that a flow dissolve is not homogenous. Flow speed is higher for each section of the heat exchanger on a wall located towards an outlet of the heat exchanger. The opposed wall is compassed by fluid with less velocity. This incident causes, that a wall of heat exchanger and area of pipes assembled to these walls are more exposed to an ash deposition. The same aspect occurs for exterior walls of the heat exchanger when a fluid flow direction is changing to the opposite.

On figure 4 is presented a temperature field of exhaust gases for two neighbouring sections of the designed heat exchanger.

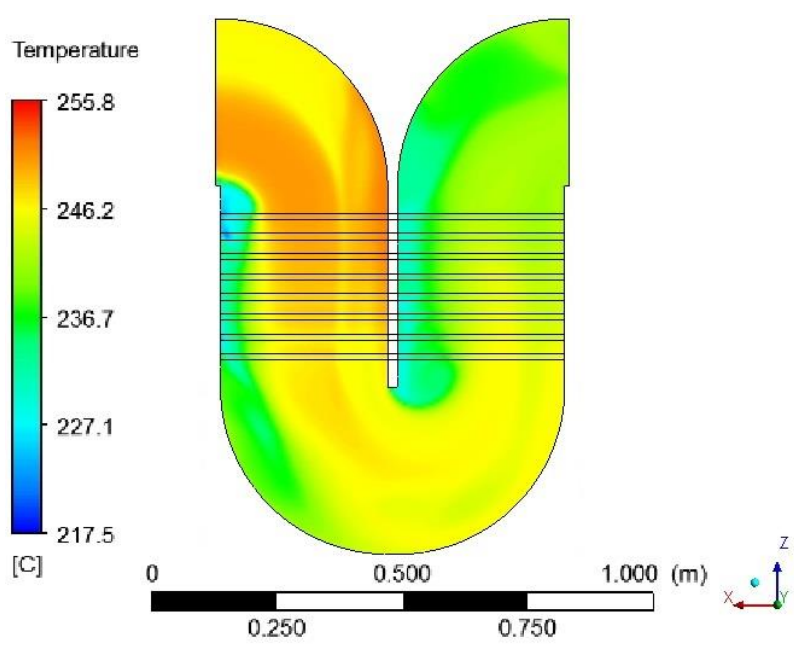

Fig. 4. Temperature distribution in two sections of the first module of the designed heat exchanger.

Realized model shows, that exhaust gases, which are flowing near left walls of each section of heat exchanger have a lower temperature than gases on the opposite side. It means, that a decreasing of temperature to water or acids liquefaction from exhaust gases will first occur on this side of heat exchanger section. It is significant to control a surfaces condition of the heat exchanger in this places during a maintenance. This operation allows preventing a heat exchanger against corrosion.

Figure 5 shows a velocity distribution for exhaust gas of a two neighboring sections.

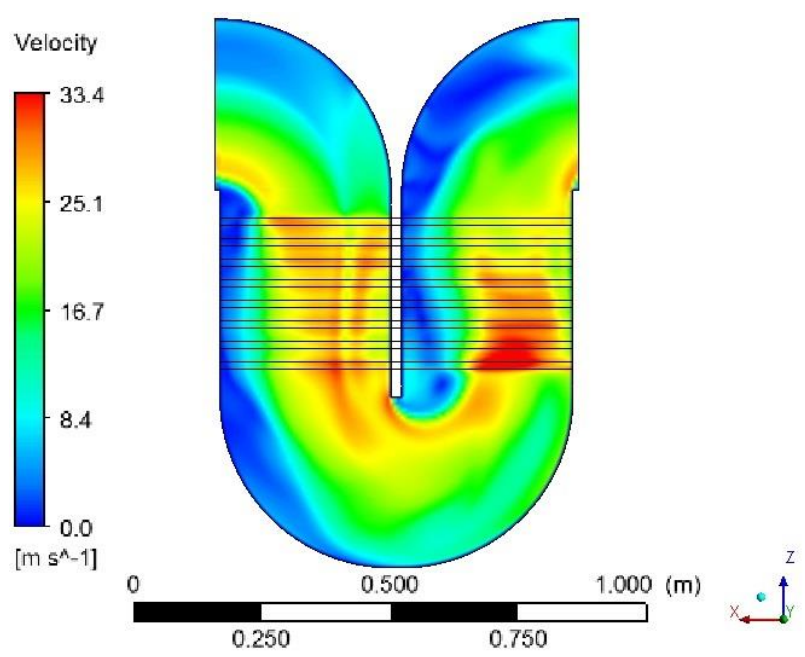

Fig. 5. Velocity distribution in two sections of the first module of the designed heat exchanger.

Velocity distribution of a fluid flow obtained during a numerical analysis in a designed heat exchanger shows, that flow inside sections of the heat exchanger is not identical for a whole volume of arranged construction. A numerical model of a smaller part of heat exchanger shows distinctly locations of detachments of a stream flow and locations of the vortex flow. The flow of exhaust gases inside the heat exchanger has a complex character. Change of direction of fluid flow causes a vortexes generation in specific parts of sections of the heat exchanger. Swirls are generated in the vertexes, where two sections of the heat exchanger are assembled together. The region of flow stagnation covers an area of intensive near left walls of heat exchanger sections. where the center of swirl is located.

The flow of exhaust gases between rows of pipes gives a rise of the velocity of a fluid because a crosssection of the heat exchanger is smaller in this place than in the area of inverting an exhaust gases direction. Numerical analysis shows, that flow of exhaust gases around the first row of pipes generate intense increasing of the velocity of a fluid, but this value is stabilized after flow around next rows of pipes. It is due to decreasing an area of fluid flow stagnation for exhaust gases, after inverting the direction of a flow and a passing an area of vortex generation. Therefore, a velocity distribution of a flow is stabilized.

\section{Conclusions}

Realized analytical and numerical calculations allowed to show the character of a work for a simple construction of heat exchanger for a food industrial plants. Analytical calculations allowed to predict a design of a heat exchanger geometry. It was done by basic calculations implementation, which is associated with a heat transfer 
theory. Analytical calculations allow for more intense analysis about a heat transfer process than a simple approach, which is presented in the paper. Information, about more advanced calculations, focused on outlet temperature and dynamics of processes for heat exchangers design can be realized according to papers [7] and [8].

Realized numerical calculations verified analytical calculations and covered the result with acceptable deviation. Numerical analysis showed, that an exhaust gasses flow into subsequent sections of analyzed construction is not homogenous. Realized research showed an area of a flow stagnation and places of vortex generation. During numerical calculations, was showed an area of decreasing a temperature, which is associated with the possibility of corrosion in these places, when a heat exchanger will be incorrectly used.

Numerical calculations are a good solution for a heat transfer analysis not only in heat exchangers modeling. This method will also help for different heat devices modeling, which is popularly used. Paper [9] presents an approach of heat transfer numerical modeling in solid fuel low power heating boilers, which are popularly used in Polish households. Numerical analysis implementation into a designing process also will help for a construction improvement of these heating devices.

\section{Acknowledgements}

The research was financed by the Poznan University of Technology financial resources for statutory activity.

\section{References}

1. I. Afonso, L. Hes, J. Maia, L. Melo, J. of Food Engineering, 57, 179-187 (2003)

2. J. Jang, L. Chen, Int. J. Heat Mass Transfer, 40, 16, 3981-3990 (1996)

3. S. Kruczek, Heating boilers - constructions and designing, (Ofic. Wyd. Pol. Wroc., Wrocław, 2001)

4. W. Gogół, Heat transfer - tables and graphs, (Wyd. Pol. War., Warszawa, 1976)

5. http://www.lipico.com/technical_references_palm_o il_properties.html, (date of available: 10.01.2018)

6. S. Wiśniewski, T. Wiśniewski, Heat transfer, (Wyd. N.-Tech., Warszawa, 2000)

7. D. Taler, Dynamic of pipes heat exchangers, (Ucz. Wydaw. Nauk.-Dydakt. AGH, Kraków, 2009)

8. D. Taler, A. Sury, Mathematical models of crossflow tube heat exchangers and their application to control of fluid outlet temperature, (Wydaw. Pol. Krak., Kraków, 2013)

9. R. Urbaniak, J. Bartoszewicz, W. Judt, Heat Trans. Res, 49, 7, 675-683 (2018) 